\title{
DERECHO Y POLÍTICAS AMBIENTALES EN GALICIA (SEGUNDO SEMESTRE 2020)
}

\author{
BELTRÁN PUENTES CoCIÑA \\ Investigador predoctoral \\ Universidade de Santiago de Compostela
}


SUMARIO: 1. Introducción. 2. Aprobación del Reglamento de protección del paisaje. 3. Inicio de la tramitación parlamentaria de la nueva Ley de ordenación del territorio. 4. La gestión de la pandemia en el plano ambiental. 5. Bibliografía.

\section{INTRODUCCIÓN}

El último semestre no ha sido época de grandes novedades en el terreno jurídicopolítico ambiental. Por una parte, no ha tocado aprobar la ley de acompañamiento de los presupuestos, que es el instrumento socorrido por el legislador gallego para hacer barridos normativos de forma anual, sin debate público y con escaso coste político. A ello se ha sumado el impacto de la pandemia en el ya habitualmente pausado ritmo de la Administración ambiental gallega, por lo que las novedades se han reducido al mínimo y las políticas de medio ambiente en Galicia parece que han entrado en un nuevo período de hibernación. Sólo una novedad merece cierta atención en el período de abril a octubre de 2020: la aprobación del Reglamento de protección del paisaje de Galicia, al que dedicaremos el apartado 2. A continuación, centraremos el apartado 3 en el relato del inicio de la tramitación parlamentaria de la nueva Ley de ordenación del territorio de Galicia, norma que deberá ser objeto de un ulterior análisis cuando se proceda a la adopción del texto definitivo. Para finalizar, dedicaremos un epígrafe a la observación de cómo la gestión de la pandemia ha afectado a la protección del medio ambiente en Galicia (apartado 4).

Como comentario previo, aunque no vaya a ser objeto de mayor análisis en esta breve crónica, nos parece interesante señalar cómo la inactividad de la Administración autonómica en materia ambiental sigue destapando viejos problemas que se han ido acumulando a lo largo del tiempo. Es el caso de la falta de prevención ante los incendios forestales que asolan cada año las mismas zonas del territorio gallego ${ }^{1} \mathrm{o}$ los reiterados supuestos de vertidos fluviales y marítimos incontrolados —últimamente

\footnotetext{
${ }^{1}$ El ingeniero forestal Fernando Rodríguez Jiménez ha estudiado para su Trabajo de Fin de Grado esta repetición en la localización de los incendios, centrando su análisis en el notable número de incendios y hectáreas quemadas en el Macizo Central Ourensán, tal y como relata el diario online Praza Pública en esta noticia del 23 de septiembre de 2020. <https://praza.gal/acontece/o-macizo-central-ourensanlume-sobre-lume-nun-grupo-de-parroquias-que-arden-cada-poucos-anos> [consulta: 17.11.2020].
} 
en el río Eume ${ }^{2}$-, problemas que ponen de manifiesto cómo la falta de efectividad de las políticas ambientales puede poner en grave peligro tanto el medio ambiente como la salud de la población gallega.

\section{APROBACIÓN DEL REGLAMENTO DE PROTECCIÓN DEL PAISAJE}

La única novedad normativa relevante en el último semestre ha sido la aprobación del Reglamento de protección del paisaje de Galicia, a través del Decreto 96/2020, de 29 de mayo, por el que se aprueba el Reglamento de la Ley 7/2008, de 7 de julio, de protección del paisaje de Galicia. La norma, comentada por DuRÁ Alemañ (2020), supone el desarrollo reglamentario la Ley de protección del paisaje doce años después de su aprobación. Esta demora es criticable si tenemos en cuenta que la propia ley fijaba un plazo de seis meses para la adopción de las oportunas disposiciones reglamentarias.

El reglamento consta de 50 artículos repartidos en 6 capítulos, a los que se les suman una disposición adicional, dos disposiciones transitorias, una disposición final y un anexo con definiciones.

El capítulo I define el objeto del reglamento que, como hemos dicho, no es otro que el desarrollo de la Ley de protección del paisaje, y determina su ámbito de aplicación. No debemos identificar la noción de paisaje con las zonas naturales o rurales, pues la legislación paisajística se aplica sobre todo el territorio de la Comunidad Autónoma zonas naturales, rurales, urbanas y periurbanas - con independencia de su estado de conservación o su nivel de degradación.

El capítulo ll se encarga de definir las competencias administrativas sobre las políticas públicas en materia de paisaje, así como de regular su integración con las políticas de ordenación territorial y sectoriales. Se regulan también los procesos de seguimiento e informe sobre el estado del paisaje en Galicia, la articulación del deber de publicidad y el derecho de acceso a la información paisajística.

El capítulo III desarrolla los instrumentos para la protección, gestión y ordenación del paisaje, esto es: los catálogos del paisaje, las directrices del paisaje, los planes de acción del paisaje y los estudios de impacto e integración paisajística de proyectos.

\footnotetext{
${ }^{2}$ Pontedeume prohíbe el consumo de agua por vertidos al río Eume. La Opinión A Coruña. 11.10.2020. $<$ https://www.laopinioncoruna.es/galicia/2020/10/11/pontedeume-prohibe-consumo-aguavertidos/1541531.html> [consulta: 17.11.2020].
} 
En primer lugar, se contemplan las funciones y los procedimientos de elaboración de los instrumentos con carácter general, así como la participación pública y los derechos de los ciudadanos en relación con la participación pública en estos instrumentos. A continuación, se disponen las previsiones específicas para cada uno de ellos (objeto, contenido, documentación, ejecución, eficacia, ámbito territorial, relación con otros instrumentos, seguimiento, etc.).

El capítulo IV desgrana los instrumentos de integración de las políticas de paisaje en las políticas territoriales y sectoriales (consideración del paisaje en los instrumentos de ordenación territorial y urbanísticas; requisitos de evaluación paisajística de planes y programas; contenido y alcance de los informes paisajísticos, etc.) mientras que el capítulo $\mathrm{V}$ detalla los organismos y los mecanismos de colaboración autonómicos para la coordinación y organización de las políticas paisajísticas, que se realizarán a través del Consello Asesor da Paisaxe y haciendo uso de pactos y acuerdos en materia de paisaje. Por último, el capítulo VI se refiere a la formación, sensibilización y educación en el ámbito paisajístico.

\section{INICIO DE LA TRAMITACIÓN PARLAMENTARIA DE LA NUEVA LEY DE ORDENACIÓN DEL TERRITORIO}

En ejercicios anteriores ya advertíamos (PUENTES, 2019) del comienzo de los trámites parlamentarios de la nueva Ley de ordenación del territorio de Galicia, cuyo primer proyecto se publicó para información pública el 6 de septiembre de 2018. La disolución del Parlamento autonómico por la celebración de elecciones en el primer semestre de 2020 hizo decaer la iniciativa legislativa, debiendo retomarse recientemente la tramitación parlamentaria (la Mesa del Parlamento admitió el proyecto a trámite el día 29 de septiembre, declarando asimismo la apertura del plazo para la presentación de enmiendas) ${ }^{3}$.

El texto ${ }^{4}$ promovido por la Dirección General de Ordenación del Territorio y Urbanismo sustituirá a la Ley 10/1995, de 23 de noviembre, de ordenación del territorio de Galicia,

\footnotetext{
${ }^{3}$ Boletín Oficial do Parlamento de Galicia, XI legislatura, n.ำ 23, 30 de septiembre de 2020, fascículo 1.

${ }^{4} \mathrm{El}$ anteproyecto de Ley de ordenación del territorio de Galicia se puede consultar en el siguiente enlace: $\quad$ https://transparencia.xunta.gal/tema/informacion-de-relevancia-xuridica/normativa-entramitacion/aprobada-e-publicada-no-dog/-/nt/0341/anteproxecto-lei-ordenacion-territorio-galicia [consulta: 17.11.2020].
} 
veinticinco años después de la aprobación. El leitmotiv de la reforma no es otro que la agilización y simplificación de los trámites administrativos para el impulso de proyectos inversores en el territorio gallego, como ha reconocido el propio Gobierno autonómico y la consejera de Medio Ambiente y Territorio en los momentos de presentación y defensa parlamentaria de la norma. En esta línea de liberalización, simplificación administrativa y eliminación de garantías ambientales para la promoción de todo tipo de intereses privados y mercantiles se inscriben otras normas anteriores como la Ley 2/2016 del suelo de Galicia -y sus repetidas modificaciones-, la Ley 5/2017 de fomento de la implantación de iniciativas empresariales o las diferentes leyes de medidas fiscales y administrativas aprobadas como acompañamiento de los presupuestos en los últimos años, que han sido objeto de comentario en pasadas ocasiones (NogueIRA \& PUENTES, 2019 y 2020).

Entre los cambios más relevantes que propone la nueva ley territorial —siempre según el texto del proyecto normativo que conocemos hasta ahora, que lógicamente podrá ser objeto de modificación y enmienda parlamentaria, por lo que dejamos para futuros trabajos un análisis más pormenorizado- destaca la introducción de los proyectos de interés autonómico (PIA) como instrumento de planificación territorial (artículos 45-62 del proyecto de ley).

Los PIA sustituyen a los planes y proyectos sectoriales de incidencia supramunicipal y se configuran como instrumentos de intervención directa en el territorio con carácter ejecutivo. Es decir, se trata de una especie de proyectos 'omnipotentes', directamente aplicables, que permitirán al Ejecutivo autonómico actuar con carácter inmediato en todo el territorio -en cualquier tipo de suelo— para implantar dotaciones urbanísticas, infraestructuras o actividades industriales. Se podrán aprobar sin apenas intervención de las entidades locales -más allá de las consultas previstas a las administraciones afectadas - y al margen del planeamiento urbanístico municipal —precisamente la imposibilidad de tramitar el proyecto según el plan vigente es uno de los supuestos para los que se plantean este tipo de proyectos-.

Los PIA pueden estar previstos en los planes sectoriales o no, pueden ser promovidos por iniciativa pública o privada -en cuyo caso bastará con la aceptación de los propietarios del $50 \%$ de la superficie total del ámbito de actuación del proyecto-y no es preciso que se sometan al régimen ordinario de la evaluación ambiental - 
solamente aquellos proyectos que afecten a espacios de la Red Natura 2000; el resto podrán ser objeto de evaluación simplificada, según lo previsto en el artículo 21 En definitiva, a modo de resumen —dado el carácter provisional de este primer análisis de urgencia-, bastará con una simple declaración de interés autonómico por parte del Consejo de la Xunta, a petición de la consejería competente por razón de materia, para realizar modificaciones en el planeamiento urbanístico municipal, proceder a la reclasificación y recalificación del suelo y determinar el emplazamiento de importantes infraestructuras, equipamientos de gran envergadura y actividades industriales.

Además de los PIA, se contemplan otros tres tipos de instrumentos de ordenación del territorio, ya existentes en la ley actualmente vigente: las directrices de ordenación del territorio (artículos 22-30 del anteproyecto), los planes territoriales integrados (arts. 3137) y los planes sectoriales (arts. 38-44). Desaparecen los programas coordinados de actuación y los planes de ordenación del medio físico. Con respecto a estos últimos, la disposición transitoria cuarta del proyecto de ley prevé que cuando sea oportuna su elaboración —según las vigentes directrices de ordenación del territorio— podrán ser sustituidos por un plan territorial integrado, un plan sectorial o una de las figuras previstas en la legislación vigente en materia de espacios naturales -esto es: planes de ordenación de los recursos naturales, planes rectores de uso y gestión, planes de gestión, normas de gestión y conservación-.

Sorprende esta remisión a la normativa de patrimonio natural cuando la mayoría de los espacios protegidos en Galicia siguen sin contar con los preceptivos instrumentos de ordenación. Aunque se han aprobado recientemente varios planes rectores de uso y gestión (Parque Nacional Marítimo-Terrestre das Illas Atlánticas, en diciembre de 2018; Parques Naturales Serra da Enciña da Lastra ${ }^{5}$ y Invernadoiro ${ }^{6}$, ambos en julio de 2019; y Monte Aloia ${ }^{7}$, en enero de 2020) todavía no cuentan con instrumentos de

\footnotetext{
${ }^{5}$ Decreto $101 / 2019$, de 11 de julio, por el que se modifica el Decreto $77 / 2002$, de 28 de febrero, por el que se aprueba el Plan de ordenación de los recursos naturales de la Serra da Enciña da Lastra y por el que se aprueba el Plan rector de uso y gestión del Parque Natural Serra da Enciña da Lastra.

${ }^{6}$ Decreto 102/2019, de 11 de julio, por el que se modifica el Decreto 166/1999, de 27 de mayo, por el que se aprueba el Plan de ordenación de los recursos naturales del Parque Natural do Invernadeiro, y por el que se aprueba el Plan rector de uso y gestión del Parque Natural do Invernadeiro.

7 DECRETO 24/2020, de 9 de enero, por el que se modifica el Decreto 274/2001, de 27 de septiembre, por el que se aprueba el Plan de ordenación de los recursos naturales del Parque Natural del Monte Aloia y por el que se aprueba el II Plan rector de uso y gestión del Parque Natural del Monte Aloia.
} 
ordenación y gestión tres parques naturales y los espacios naturales amparados por otras figuras de protección.

En este sentido, también se echa en falta un papel más relevante para los espacios naturales protegidos a la hora de planificar la ordenación del territorio, habida cuenta de que Galicia cuenta con una red de espacios naturales protegidos muy pequeña (es la comunidad autónoma con menor porcentaje de superficie protegida por la Red Natura 2000) y en consonancia con las últimas directrices europeas ${ }^{8}$. En este sentido, URIARTE (2020) señala la necesidad de extender la infraestructura verde y penetrar en la estructura territorial, afirmando que "es necesario que su diseño se incorpore en los instrumentos de planeamiento, inicialmente en los de naturaleza territorial, para expandirse gradualmente a los urbanísticos y a la planificación sectorial correspondiente". En particular, la autora aplaude la actualización de las Directrices de Ordenación del Territorio que ha llevado a cabo el Gobierno del País Vasco de manera reciente, destacando la apuesta por la integración del enfoque de los servicios ecosistémitcos en la ordenación territorial, junto al compromiso de desarrollar una infraestructura verde en todas las escalas (URIARTE, 2020, p. 39).

Por otra parte, volviendo al proyecto normativo del Gobierno gallego, se excluyen del ámbito de actuación de la nueva Ley de ordenación del territorio los planes sectoriales y proyectos de interés autonómico que tenga por objeto la implantación y gestión de parques empresariales y parques eólicos. Según dispone la disposición adicional quinta, estos proyectos se regularán por las disposiciones de la Ley $5 / 2017$, de fomento de la implantación de iniciativas empresariales en Galicia.

En cuanto a las enmiendas parlamentarias, el Grupo Parlamentario Popular de Galicia ha introducido alguna enmienda que ya ha sido aprobada en la Cámara autonómica, como la creación del Consejo de Ordenación del Territorio de Galicia (nuevo artículo 25 bis) como órgano colegiado de carácter consultivo, adscrito a la consejería competente en materia de territorio y competente para la coordinación de los intereses territoriales, públicos y privados que afecten al territorio de la comunidad. Este órgano ostentaría las funciones de informar los instrumentos de ordenación territorial y los restantes planes, programas y proyectos autonómicos que incidan en la ordenación

\footnotetext{
${ }^{8}$ Comunicación de la Comisión al Parlamento Europeo, al Consejo, al CESE y al CDR. Infraestructura verde: mejora del capital natural de Europa. COM (2013) 249 final.
} 
del territorio; evacuar consultas que le sean requeridas por el Parlamento o la persona responsable de la consejería; promover la realización de investigaciones, reuniones científicas, estudios y actuaciones de divulgación en materia de ordenación del territorio; e informar a la Comisión Superior de Urbanismo en aquellas cuestiones que, por su dimensión o importancia, lo requieran a solicitud de su presidente.

Los dos grupos de la oposición han presentado enmiendas a la totalidad que han sido rechazadas por la mayoría popular en la Cámara. Entre otras cuestiones, los socialistas alegan que el proyecto de ley es una cortina de humo en la que esconder la falta de gestión del Ejecutivo, que no quedan acreditados los motivos y la necesidad de una nueva ley, que confunde la flexibilidad con la liberalización y que se lesiona el principio de autonomía municipal ${ }^{9}$. Por su parte, los nacionalistas señalan que la ley del 95 tuvo un impacto limitado como consecuencia de una producción normativa de desarrollo muy escasa, que solamente han proliferado instrumentos de menor escala que responden a intereses particulares frente a la planificación de conjunto y el interés general y que en esta línea se inscribe la aprobación de los PIA, en el marco de la estrategia trazada por la ley de fomento de las iniciativas empresariales ${ }^{10}$.

\section{LA GESTIÓN DE LA PANDEMIA EN EL PLANO AMBIENTAL}

Por una parte, todo parecía indicar que el confinamiento obligado por la crisis sanitaria del Covid-19 iba a suponer un respiro para el medio ambiente. Así se hizo notar en los niveles de emisiones registrados en los espacios urbanos ${ }^{11}$ o en aquellos espacios naturales en los que la presión turística impide el normal desarrollo de la biodiversidad. Sin embargo, pronto se conocieron algunas decisiones controvertidas en el plano ambiental.

En este sentido, una de las decisiones más discutidas fue la reanudación de los procedimientos administrativos para la tramitación de proyectos de impacto ambiental,

\footnotetext{
${ }^{9}$ Enmienda a la totalidad presentada por el Grupo Parlamentario dos Socialistas de Galicia, núm. de registro 3814, 19/10/2020. En Boletín Oficial del Parlamento de Galicia, XI legislatura, núm. 36, 28/10/2020, fascículo 3.

${ }^{10}$ Enmienda a la totalidad presentada por el Grupo Parlamentario do Bloque Nacionalista Galego, núm. de registro 3832, 19/10/2020. En Boletín Oficial del Parlamento de Galicia, XI legislatura, núm. 36, 28/20/2020, fascículo 3.

${ }^{11}$ La polución del aire se redujo en más de la mitad en casi todas las ciudades gallegas durante el estado de alarma. $<$ http://adega.gal/novas.php?id=981\&idioma=gl\&sec=209> [consulta: 17.11.2020].
} 
cuyos trámites de obtención de permisos, autorizaciones o licencias y evaluación ambiental habían sido suspendidos por el Real Decreto 463/2020, de 14 de marzo, por el que se declara el estado de alarma ${ }^{12}$. La Consellería de Medio Ambiente, Territorio e Vivenda decidió la continuación de los plazos a través de la Orden de 27 de abril de $2020^{13}$, estando todavía vigente el estado de alarma y por tanto encontrándose la población en situación de confinamiento domiciliario. Esta circunstancia pudo suponer una dificultad añadida para el acceso a la información ambiental, la reunión de los colectivos legitimados para el control de la normativa y las políticas ambientales y en definitiva para la participación en los asuntos públicos de las personas y los colectivos afectados por los proyectos en trámite. La orden de la Consellería se basó en una cláusula del Real Decreto de estado de alarma por la que se podría acordar la continuación de los procedimientos administrativos en determinados casos. En particular, el apartado cuatro de la disposición adicional permitía la continuación de los procedimientos que fuesen indispensables para la protección del interés general o para el funcionamiento básico de los servicios. Con esta base, la Consellería consideró que la tramitación de expedientes administrativos, la emisión de informes y la concesión de permisos y licencias era un medio necesario para la reactivación de la actividad económica, identificado por tanto el interés general con intereses económicos particulares.

Esta decisión contrasta con otras de signo contrario en ámbitos que sí podrían tener una repercusión positiva en términos ambientales, como el cierre preventivo de los mercados de proximidad en los que se produce la venta directa de productos agrícolas y ganaderos a través de la Orden de la Consellería de Medio Rural del 23 de marzo de $2020^{14}$.

\footnotetext{
12 Real Decreto 463/2020, de 14 de marzo, por el que se declara el estado de alarma para la gestión de la situación de crisis sanitaria ocasionada por el COVID-19.

${ }^{13}$ ORDEN de 27 de abril de 2020 de la Consellería de Medio Ambiente, Territorio y Vivienda por la que se acuerda el inicio y/o la continuación de la tramitación de determinados procedimientos indispensables para la protección del interés general o para el funcionamiento básico de los servicios públicos en el ámbito de esta consellería durante la vigencia del estado de alarma.

${ }^{14}$ Orden de 23 de marzo de 2020 por la que adoptan medidas de carácter obligatorio en relación con el COVID-19 en cumplimiento del Acuerdo del Centro de Coordinación Operativa de la emergencia sanitaria en la Comunidad Autónoma de Galicia (Cecop), de 18 de marzo de 2020, sobre la venta directa de productos agroganaderos en los mercados, la venta de productos vegetales para la plantación en huertas de consumidores finales y el desplazamiento de agricultores y viticultores a los efectos de la realización de actividades agrarias.
} 


\section{BIBLIOGRAFÍA}

DuRÁ Alemañ, C. J. (2020), "Legislación al día. Galicia. Paisaje”, en Actualidad Jurídica Ambiental, https://www.actualidadjuridicaambiental.com/legislacion-al-diagalicia-paisaje/ [consulta: 17.10.2020].

Nogueira López, A. \& Puentes CociñA, B. (2019), "Galicia: un latido ambiental autonómico en mínimos busca que los ayuntamientos hagan los deberes", en Observatorio de Políticas Ambientales 2019. Madrid: CIEMAT.

Nogueira López, A. \& Puentes CociñA, B. (2020), "Galicia: la Xunta se reactiva in extremis sin abandonar la pulsión desreguladora", en Observatorio de Políticas Ambientales 2020. Madrid: CIEMAT.

Puentes CociñA, B. (2019), "Derecho y políticas ambientales en Galicia (primer semestre 2019)", en Revista Catalana de Dret Ambiental, vol. 10, n. 1, https://revistes.urv.cat/index.php/rcda/article/view/2543 [consulta 13.11.2020].

URIARTE RICOTE, M. (2020), "El valor ambiental de la infraestructura verde en el nuevo modelo vasco de ordenación del territorio", en Actualidad Jurídica Ambiental, https://www.actualidadjuridicaambiental.com/articulo-doctrinal-el-valorambiental-de-la-infraestructura-verde-en-el-nuevo-modelo-vasco-deordenacion-del-territorio/ [consulta: 17.11.2020]. 